\title{
Total Skin Electron Irradiation (TSEI) May Induce Systemic Progression of Mycosis Fungoides (MF) - Cutaneous T-Cell Lymphoma (CTCL)
}

\author{
Sherif G Shaaban ${ }^{1}$, Mohammed K Khan ${ }^{2}$, Natasha Savage ${ }^{1}$, Weisi Yan ${ }^{1}$, Mahboubeh Pishgou ${ }^{1}$, Loretta \\ Davis $^{1}$ and Waleed F Mourad ${ }^{1 *}$
}

${ }^{1}$ Medical College of Georgia, Augusta University, GA, USA

${ }^{2}$ Emory University, Atlanta, GA, USA

\author{
Keywords \\ TSEI, Mycosis fungoides, Radiation therapy
}

The management of MF-CTCL is based on a "stage-based" approach and an evaluation by a multidisciplinary team is preferred. Initial treatment in patients with local (patch/ plaque) disease includes skin-directed therapies (localized or generalized), with the addition of systemic biologic therapy for refractory, or progressive disease. In patients with unfavorable prognostic features (e.g., folliculotropic or large-cell transformed MF, or B1 involvement) systemic biologic therapies may be introduced earlier in the treatment algorithm. Patients who do not respond to biologic therapy or those with very aggressive or extracutaneous disease may be treated with chemotherapy [1-3].

TSEI represents an integral part in the treatment of advanced MF. TSEI is characterized by a favorable local toxicity profile with excellent response rates. The impact of TSEI on systemic progression remains unclear. A 75-YOAAM with stage IIB Cutaneous T-Cell Lymphoma had clinically indolent disease for many years. He then presented with multiple cutaneous lesions with increasing evolution of his disease. He received TSEI to 18 Gy in 12 fractions followed by additional boost to many sites. One month later, he presented with diffuse neck swelling, difficulty breathing, and hoarseness. $\mathrm{PET} / \mathrm{CT}$ scan revealed diffuse systemic progression. Despite salvage radiation (RT) and chemotherapy, his disease quickly progressed further and was ultimately fatal. TSEI's impact on systemic progression of MF merits further investigation, especially in the context of clinicopathological evaluation of MF transformation and the potential value of combining novel immunotherapeutic agents like checkpoint inhibitors with RT to potentially further improve outcomes for such patients with aggressive transformation MFCTCL.

\section{Introduction}

Cutaneous T-cell lymphomas (CTCLs) comprise $70 \%$ of all primary cutaneous lymphomas with an estimated annual incidence of 0.3-1 per 100,000 people in the United States of America [4,5]. Mycosis fungoides (MF) is the most common type of CTCLs, accounting for about $50-70 \%$ of cases. About $85 \%$ of patients present with early stage disease and have an indolent course with slow progression or remain asymptomatic during their life [1]. The presence of lymphadenopathy, involvement of peripheral blood, and visceral disease adversely affects prognosis [6]. Current treatment options are "stage-based", including skin-directed therapies and localized radiation treatment (RT) for early stages and systemic therapy combined with total skin electron irradiation (TSEI) for more advanced stages. Sequential therapies are selected based on availability and convenience for the patient, as well as on short- and long-term toxicity profiles $[2,3,7,8]$. Historically, TSEI represents a treatment option that has a vital role in the treatment of MF, with mild and reversible treatment related side effects [9]. Herein, we describe a unique case of CTCL who developed fatal rapid systemic progression shortly after treatment with TSEI, aiming to highlight the potential negative impact of TSEI on systemic progression of MF and explore the future directives of prospective value of combining novel immunotherapeutic agents like checkpoint inhibitors with RT to potentially further improve outcomes for such patients with aggressive transformation MF-CTCL.

*Corresponding author: Waleed $\mathrm{F}$ Mourad, MD, Medical College of Georgia, Augusta University, GA, USA, Tel: 347-782-7886

Accepted: November 17, 2018

Published online: November 19, 2018

Citation: Shaaban SG, Khan MK, Savage N, et al. (2018) Total Skin Electron Irradiation (TSEI) May Induce Systemic Progression of Mycosis Fungoides (MF) - Cutaneous T-Cell Lymphoma (CTCL). Dermatol Arch 2(1):62-67 

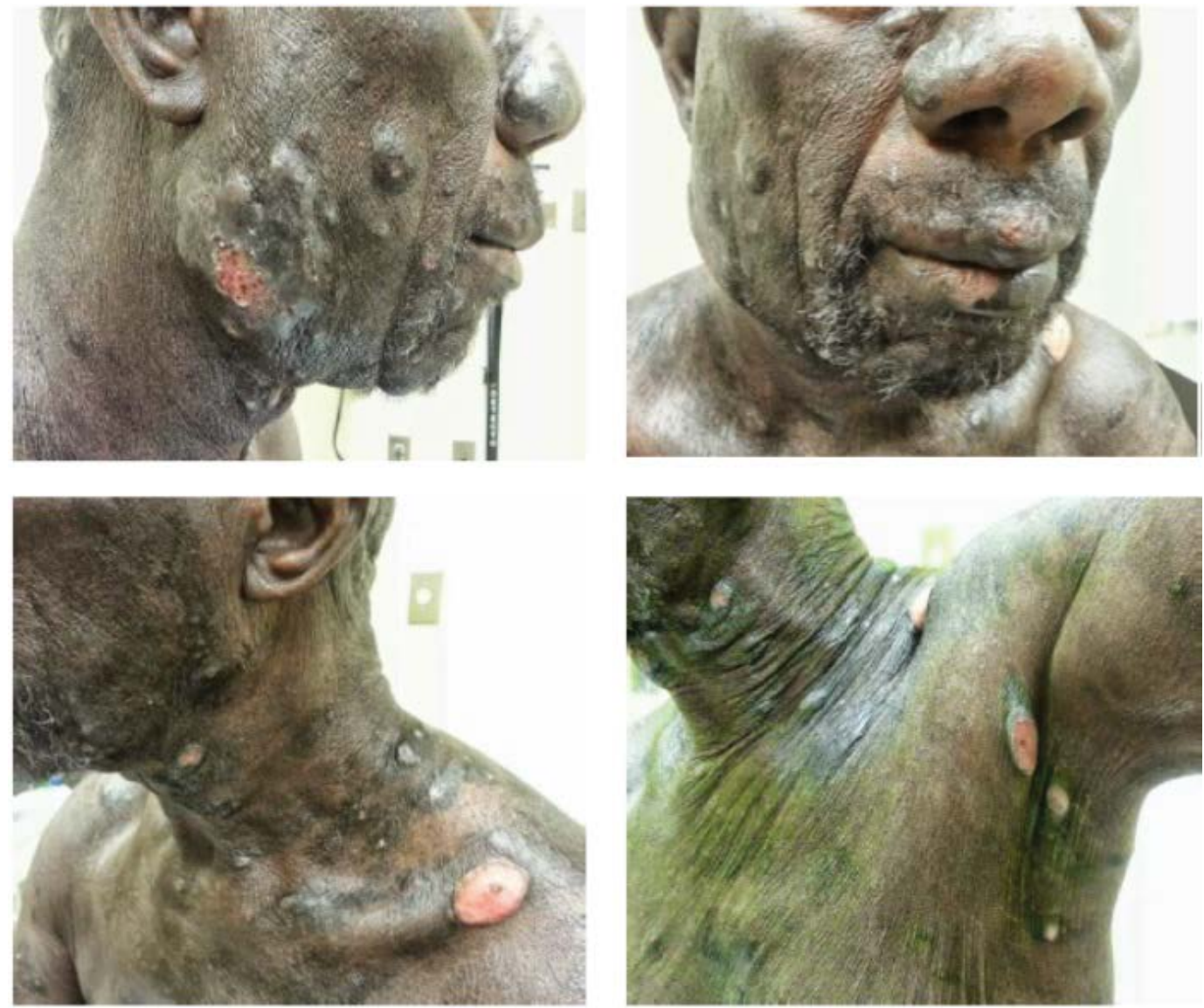

Figures 1: Multiple cutaneous lesions involving scalp, neck, and extremities.

\section{Case Report}

A 75-year-old African American male presented with multiple cutaneous lesions involving the majority of his scalp, neck, trunk and extremities (Figure 1). His condition initially started in 2003 when he noticed a cutaneous lesion on his chest. He was diagnosed with cutaneous T-cell lymphoma (CTCL), "mycosis fungoides", (MF) and received topical treatment and phototherapy. His disease had been in reasonable control on topical and phototherapies for 12 years, with a waxing and waning course. In 2015, he presented with diffuse hyperpigmented scaly skin lesions with extensive plaques and desquamated areas. His largest lesions were in the right cheek, scalp, left axilla, left back, and left supraclavicular fossa. The lesions were described as more tumor-like, deeply invasive, with ulceration and necrosis, which were starting to cause significant pain, bleeding, and itching for the patient. Thus, he was referred for radiotherapy (RT). The patient received localized electron beam radiation therapy (EBRT) to the right cheek and left supraclavicular area; each site received $20 \mathrm{~Gy}$ in 10 fractions at the rate of $200 \mathrm{cGy}$ daily fraction via $9 \mathrm{MeV}$ and $6 \mathrm{MeV}$ enface plus $5 \mathrm{~mm}$ daily bolus for the cheek and supraclavicular lesions respectively, as shown in Figure 2. During EBRT, these lesions showed marked improvement with a complete clinical response by $4^{\text {th }}$ week. Two months later, the patient received TSEI to 18 Gy in 12 fractions, followed by focal boost of $3 \mathrm{~Gy}$ in a single fraction to the right eye, $4 \mathrm{~Gy}$ in a single fraction to upper lip, $8 \mathrm{~Gy}$ in 4 fractions to the scalp, $8 \mathrm{~Gy}$ in 4 fractions to his shoulders, axilla, and perineum, and $10.5 \mathrm{~Gy}$ in 7 fractions to the soles of his feet bilaterally. The areas that were boosted were considered to have received lower doses of radiotherapy during TSEI. Thus, a "supplemental" radiotherapy boost was provided. One month later, he presented with diffuse neck swelling, difficulty breathing particularly while lying flat, and hoarseness. On examination, there was bulky palpable lymphadenopathy in bilateral cervical chains from levels I-IV. Laryngoscopy revealed hypertrophy of nasopharynx and BOT and decreased motion of left TVC. PET/CT scan (Figure 3) revealed diffuse disease progression including pharynx/ Waldeyer's ring, bilateral neck, bilateral axilla, bilateral pelvis, bilateral femoral and inguinal regions, as well as extranodal sites including spleen, mediastinum and pleura. Moreover, it showed compression fractures of T10 and T11. Excisional axillary lymph node biopsy was performed, which revealed diffuse effacement of the normal nodal architecture by a mixed inflammatory infiltrate (Figure 4A). This infiltrate was predominated by eosinophils, histiocytes and medium sized lymphoid cells with irregular nuclear contours and partially condensed chromatin. Flow cytometric analysis of the lymph node detected an aberrant T-cell population with loss of CD7 as well as aberrantly dim CD3 and CD4. PD-L1 immunohistochemical stain highlighted $70 \%$ of the neoplastic cells (Figure 4B). The patient then received another course of irradiation to his bilateral neck lymph nodes, pharynx/ Waldeyer's ring, involved salivary glands and supraclavicular 


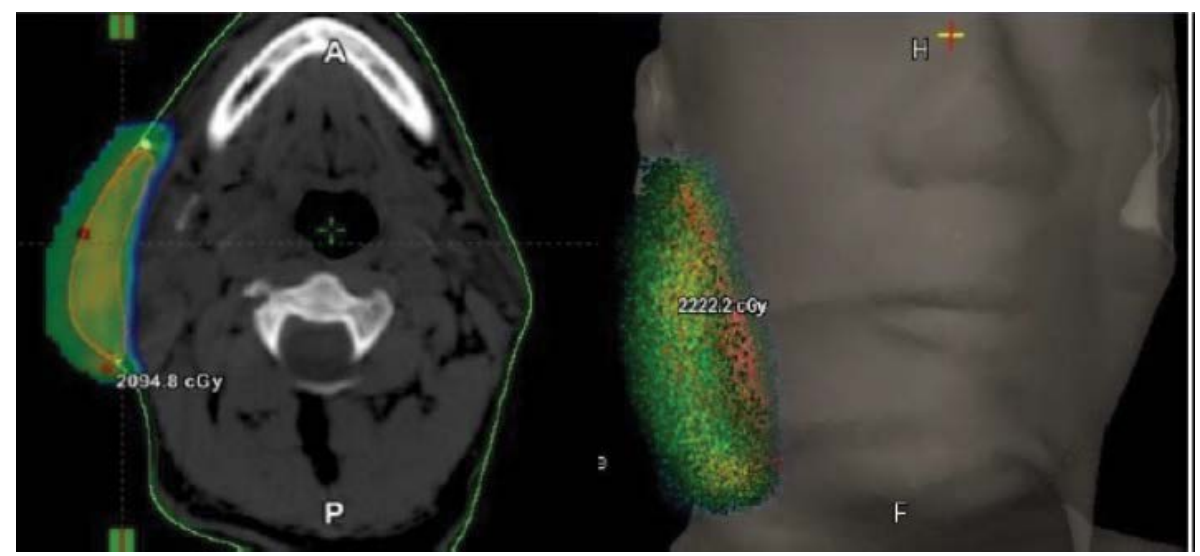

Figures 2: Right cheek enface $9 \mathrm{MeV}+5 \mathrm{~mm}$ daily bolus. Dose delivered was $20 \mathrm{~Gy}$ in 10 fractions.

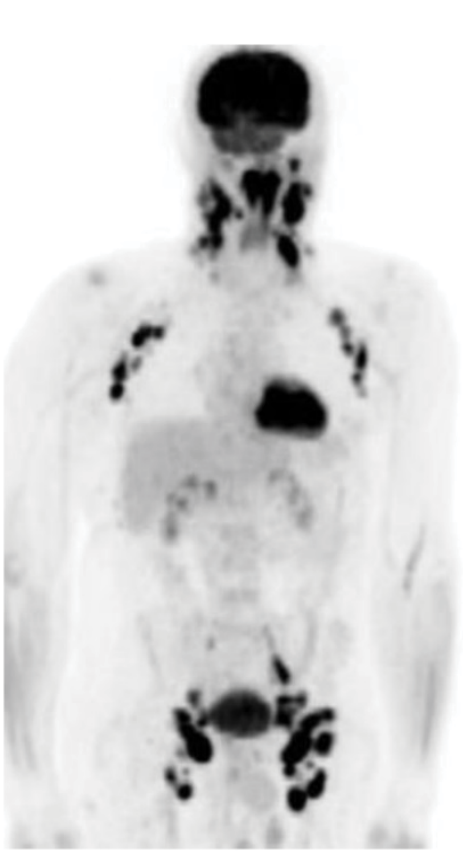

Figure 3: PET/CT scan showing diffuse disease progression. lymph nodes to $20 \mathrm{~Gy}$ in 10 fractions, aiming to relieve his obstructive symptoms and to prevent paralysis of his left TVC (Figure 5). Unfortunately, despite local improvement of his head and neck disease, he deteriorated systemically and was admitted to the hospital for hematemesis and melena. Upper endoscopy revealed diffuse gastric ulcerative disease consistent with mucosal involvement of his lymphoma. He ultimately developed severe septic shock compounded by adrenal insufficiency leading to respiratory failure and death.

\section{Discussion}

The most significant prognostic factors affecting survival in patients with MF include age at presentation, extent and type of skin involvement, overall stage, presence of extracutaneous disease, and peripheral blood involvement $[6,7]$. Kim, et al. studied the long-term outcome of 525 patients with MF and found that risk of disease progression, development of extracutaneous disease and death was correlated with initial T classification. Furthermore, they reported that the extent of blood involvement was significantly correlated with poor survival outcomes [7]. Patients with advanced stage disease (stages IIB, III, and IVA) with erythroderma and lymph node
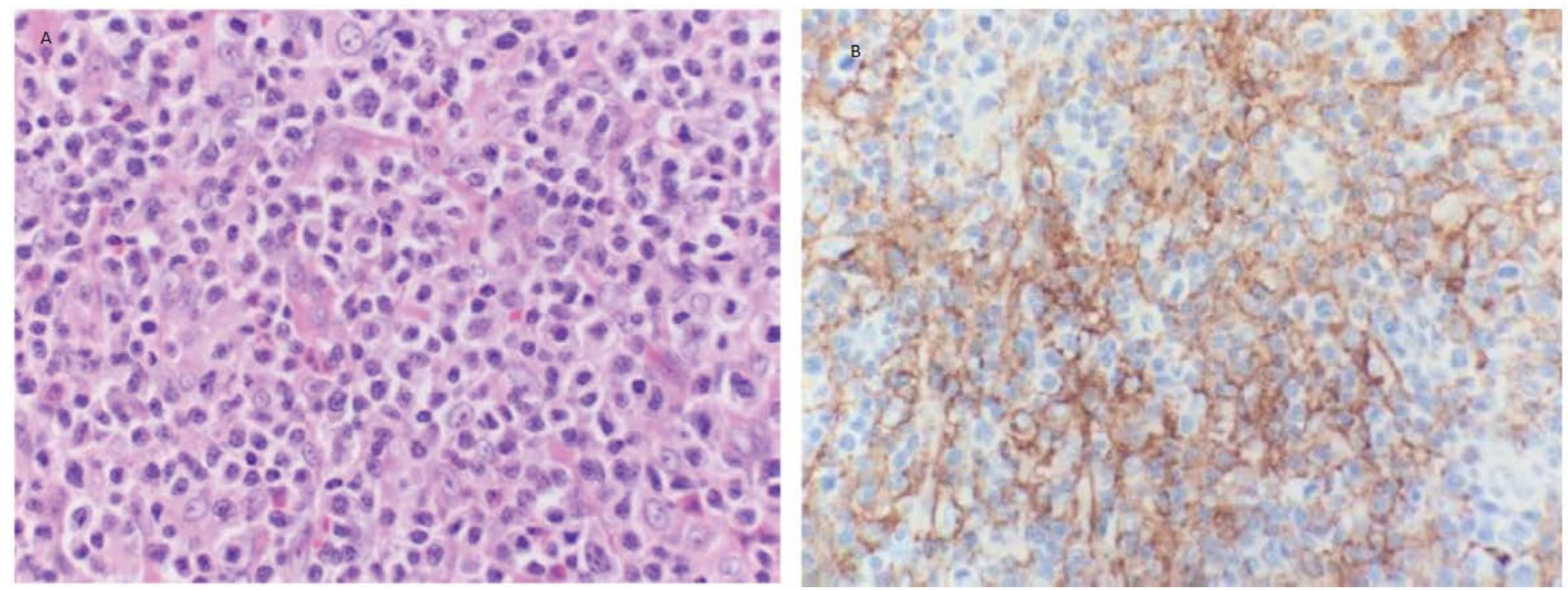

Figure 4: A) Hematoxylin and Eosin stained slide, original magnification 500X; B) Clone 22C3 Clarient Diagnostic Services, original magnification 500X. 


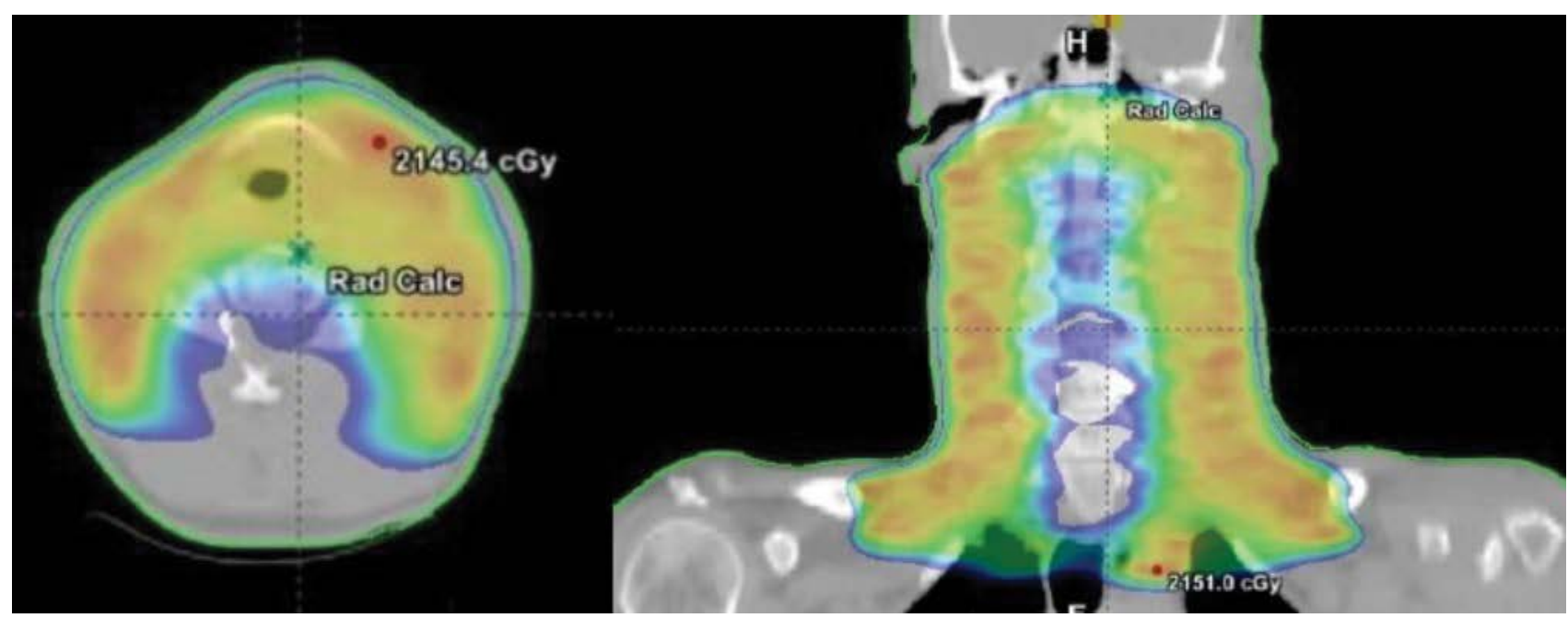

Figures 5: EBRT $20 \mathrm{~Gy}$ in 10 fractions to bilateral enlarged neck lymph nodes.

or blood involvement but no visceral involvement have a median survival of 5 years, whereas this survival decreases to be 2.5 years or less with visceral disease [8]. RT is a highly effective treatment for MF. It can be delivered to variable skin surfaces and with either palliative or curative intent. Due to the extremely radiosensitive nature of MF, high response rates can be obtained with very low doses of radiation [1]. Focal radiation treatment provides excellent local control and palliation of symptoms. Dose should be determined on the basis of potential normal tissue complication, the irradiated volume, the skin condition, prior RT to the site, and whether TSEI is considered definitive or palliative [912]. The International Lymphoma Radiation Oncology Group consensus guidelines encourage the use of doses of 20 to 24 Gy, as most patients respond to lower radiation doses [13]. TSEI represents an important treatment choice for locally advanced and refractory cases of MF. Its implementation requires a highly-experienced team to ensure adequate dose delivery to the target sites and to avoid excessive toxicity to healthy tissue [8]. It depends on the use of linear accelerators to generate electron beam directed to penetrate the skin at a limited depth $(<2 \mathrm{~cm})$ while ensuring minimal dose reaches visceral organs. A variety of techniques may be used including large electron field techniques, rotational techniques, and techniques involving patient or beam movement during irradiation [14-17]. Generally, these require treating patients in the standing position or on a rotating platform, or else achieving multiple different positions to expose all body surfaces [18,19]. Navi, et al. reviewed the Stanford University experience with TSEI and found that a dose of $30 \mathrm{~Gy}$ or greater is highly effective in treatment of T2-T3 MF with $100 \%$ overall response rate [14]. Specht, et al. considered a total treatment dose of 30-36 Gy, 4 or 5 days per week, over 8-10 weeks of TSEI in MF patients. Few studies have researched the potential value of lower dose TSEI (10-12 Gy), aiming at reduced treatment duration, fewer side effects, and the opportunity for retreatment if required [13]. Harrison and colleagues showed TSEI in doses of $10 \mathrm{~Gy}$ to $<20 \mathrm{~Gy}$ and 20
Gy to < 30 Gy were comparable to the standard dose regimen in terms of overall response and survival $[20,21]$ Moreover, Hoppe, et al. analyzed data from three clinical trials using lowdose (12 Gy) TSEI and found excellent overall response rate of $88 \%$ and complete response rate of $27 \%$ with acceptable toxicity profile [21]. Generally, TSEI side effects are well tolerated and most patients can resume their daily normal activities shortly after [22]. Treatment related toxicity is more common with the standard dose of 30-36 Gy. Patients may experience some temporary short-term side effects such as fatigue, mild to-moderate radiation dermatitis, temporary scalp alopecia, and nail stasis. Uncommon reported side effects are blisters on the fingers and feet, self-limiting anhidrosis, minor parotiditis, and Gynecomastia and infertility in males. Nevertheless, our case presented one month after TSEI completion with diffuse systemic progression, clinically symptomatic and as confirmed by PET/CT scan. The case presentation is consistent with rapid disease progression and suspected transformation into a more aggressive pathology given the previously indolent behavior for the preceding 13 years. This is a unique case. Our hypothesis is supported by time of progression, which occurred acutely after TSEI and during a period of expected disease control as typical in patients undergoing RT. Furthermore, one could surmise that the change in disease behavior may have been exacerbated by the immunosuppressive effects of RT, especially in a patient with advanced age, a known risk factor for post radiotherapy immunosuppression. Noteworthy, to date there is no evidence in the literature that TSEI has any shortor long-term adverse effects on systemic progression due to bone marrow or immune suppression [23]. Therefore, we propose a potential mechanism is via tumor immune escape through overexpression of immune checkpoint proteins such as PD-1, the PD-1 ligands, or CTLA-4 on either tumor cells or T-cells within the tumor microenvironment. Perhaps the patient would have benefited from combination therapy with RT and an immune checkpoint inhibitor, an emerging concept in immunotherapy for malignancies under current 
investigation. Radiation can potentiate the effects of PD-1 and CTLA-4 antibodies: Van-poullie Box, et al. presented the synergistic effect of radiation and CTLA-4 antibodies on tumor rejection. Some of the proposed molecular mechanisms include; increased T-cell priming, antigen shedding by radiation, antigen presentation by dendritic cells in post radiation environment, and increased effector T-cell homing via up-regulation of CXCL16 [24]. The authors argue that CTLA-4 and radiotherapy in combination will have complimentary effects that may lead to increased tumor rejection. Several clinical trials are underway to test this hypothesis in differing malignancy subtypes. Deng, et al. have demonstrated that radiation in combination with anti-PD-L1 treatment can promote increased anti-tumor immunity. The mechanisms behind such an effect is proposed to include; increased tumor cell death and antigen shedding by radiation, increased inflammation in post radiated tissue, increased PD-L1 expression in radiated tissue, increased cytotoxic actions of CD8+ T-cells, and alteration of the tumor immune microenvironment by reducing myeloid derived suppressor cells and increased T-cell infiltration. The authors concluded that PD-L1/PD-1 axis and the interactions of these antibodies with radiation helps to establish a rational design for future combination therapy trials [25]. Thus, combining the findings of the Deng, et al. with the case report by Ogunrinade, et al. it is potentially conceivable that radiation may have altered immune checkpoint protein expression in our patient allowing for increased tumor immune escape. The combination of RT with anti-PD-1 blockade may pose a better and more rationale multi-modality approach to address both skin and systemic disease [26]. It is also possible that our patient's tumor had previously acquired altered PD-1 or CTLA-4 protein expression as a mechanism of tumorigenicity. Thus, knowing this beforehand may have allowed an opportunity to treat him with a combination of an immune checkpoint inhibitor with RT to improve his outcome. Further trials are warranted to test immunotherapy in combination with RT for CTCL.

\section{Conclusion}

The impact of TSEI on systemic progression of MF merits further investigation, especially in the context of the clinicopathological evaluation of MF transformation and the value of combining novel immunotherapeutic agents such as immune checkpoint inhibitors like anti-PD-1, anti-CTLA-4, and others with RT.

\section{References}

1. Mazzeo E, Rubino L, Buglione M, et al. (2014) The current management of mycosis fungoides and Sezary syndrome and the role of radiotherapy: Principles and indications. Rep Pract Oncol Radiother 19: 77-91.

2. Hymes KB (2007) Choices in the treatment of cutaneous T-cell lymphoma. Oncology (Williston Park) 21: 18-23.

3. Rosen ST, Querfeld C (2006) Primary cutaneous T-cell lymphomas. Hematology Am Soc Hematol Educ Program 323-330.

4. Bradford PT, Devesa SS, Anderson WF, et al. (2009) Cutaneous lymphoma incidence patterns in the United States: A populationbased study of 3884 cases. Blood 113: 5064-5073.
5. Assaf C, Gellrich S, Steinhoff M, et al. (2007) Cutaneous lymphomas in Germany: An analysis of the Central Cutaneous Lymphoma Registry of the German Society of Dermatology (DDG). J Dtsch Dermatol Ges 5: 662-668.

6. Agar NS, Wedgeworth E, Crichton S, et al. (2010) Survival outcomes and prognostic factors in mycosis fungoides/Sezary syndrome: Validation of the revised International Society for Cutaneous Lymphomas/European Organisation for Research and Treatment of Cancer staging proposal. J Clin Oncol 28: 47304739.

7. Kim YH, Liu HL, Mraz-Gernhard S, et al. (2003) Long-term outcome of 525 patients with mycosis fungoides and Sezary syndrome: clinical prognostic factors and risk for disease progression. Arch Dermatol 139: 857-866.

8. Arulogun SO, Prince HM, Ng J, et al. (2008) Long-term outcomes of patients with advanced-stage cutaneous T-cell lymphoma and large cell transformation. Blood 112: 3082-3087.

9. Jones GW, Kacinski BM, Wilson LD, et al. (2002) Total skin electron radiation in the management of mycosis fungoides: Consensus of the European Organization for Research and Treatment of Cancer (EORTC) Cutaneous Lymphoma Project Group. J Am Acad Dermatol 47: 364-370.

10. Scarisbrick JJ, Kim YH, Whittaker SJ, et al. (2014) Prognostic factors, prognostic indices and staging in mycosis fungoides and Sézary syndrome: Where are we now? Br J Dermatol 170: 1226-1236.

11. Micaily B, Miyamoto C, Kantor G, et al. (1998) Radiotherapy for unilesional mycosis fungoides. Int J Radiat Oncol Biol Phys 42: 361-364.

12. Wilson LD, Kacinski BM, Jones GW (1998) Local superficial radiotherapy in the management of minimal stage IA cutaneous T-cell lymphoma (Mycosis Fungoides). Int J Radiat Oncol Biol Phys 40: 109-115.

13. Specht L, Dabaja B, Illidge T, et al. (2015) Modern radiation therapy for primary cutaneous lymphomas: Field and dose guidelines from the international lymphoma radiation oncology group. Int J Radiat Oncol Biol Phys 92: 32-39.

14. Navi D, Riaz N, Levin YS, et al. (2011) The Stanford University experience with conventional-dose, total skin electron-beam therapy in the treatment of generalized patch or plaque (T2) and tumor (T3) mycosis fungoides. Arch Dermatol 147: 561-567.

15. Evans MD, Hudon C, Podgorsak EB, et al. (2014) Institutional experience with a rotational total skin electron irradiation (RTSEI) technique three decade review (1981-2012). Rep Pract Oncol Radiother 19: 120-134.

16. Hensley FW, Major G, Edel C, et al. (2014) Technical and dosimetric aspects of the total skin electron beam technique implemented at Heidelberg University Hospital. Rep Pract Oncol Radiother 19: 135-143.

17. Kim TH, Pla C, Pla M, et al. (1984) Clinical aspects of a rotational total skin electron irradiation. Br J Radiol 57: 501-506.

18. Sewchand W, Khan FM, Williamson J (1979) Total-body superficial electron-beam therapy using a multiple-field pendulum-arc technique. Radiology 130: 493-498.

19. Wu JM, Leung SW, Wang CJ, et al. (1997) Lying-on position of total skin electron therapy. Int J Radiat Oncol Biol Phys 39: 521-528.

20. Harrison C, Young J, Navi D, et al. (2011) Revisiting low-dose total skin electron beam therapy in mycosis fungoides. Int J Radiat Oncol Biol Phys 81: e651-e657. 
21. Hoppe RT, Harrison C, Tavallaee M, et al. (2015) Low-dose total skin electron beam therapy as an effective modality to reduce disease burden in patients with mycosis fungoides: results of a pooled analysis from 3 phase-II clinical trials. J Am Acad Dermatol 72: 286-292.

22. Wilson LD (2003) Delivery and sequelae of total skin electron beam therapy. Arch Dermatol 139: 812-813.

23. Barker HE, Paget JTE, Khan AA, et al. (2015) The tumour microenvironment after radiotherapy: Mechanisms of resistance and recurrence. Nat Rev Cancer 15: 409-425.
24. Vanpouille-Boxa C, Pilones KA, Wennerberg E, et al. (2015) In situ vaccination by radiotherapy to improve responses to antiCTLA-4 treatment. Vaccine 33: 7415-7422.

25. Deng L, Liang H, Burnette B, et al. (2014) Irradiation and antiPD-L1 treatment synergistically promote antitumor immunity in mice. J Clin Invest 124: 687-695.

26. Oguninade O, Ahn CS, Gergis U, et al. (2014) Cutaneous lymphocyte antigen expression loss and PD1 positivity in early cutaneous lesions of rapidly progressive mycosis fungoides. Clin Case Rep 2: 209-218. 